J. Phys. IV France 130 (2005) 229-244

(C) EDP Sciences, Les Ulis

DOI: $10.1051 /$ jp4:2005130016

\title{
Modélisation de la dynamique de la chaîne peptidique des protéines en solution par RMN à travers les couplages dipolaires
}

\author{
G. Bouvignies, P. Bernadó et M. Blackledge \\ Institut de Biologie Structurale Jean-Pierre Ebel, UJF-CNRS-C.E.A., 41 rue Jules Horowitz, \\ 38027 Grenoble Cedex, France \\ e-mail : Martin.Blackledge@ibs.fr
}

\begin{abstract}
Résumé. L'activité d'une protéine est liée non seulement à sa structure, mais également à sa dynamique, et il est important de connaître la nature de ses mouvements pour comprendre sa fonction biologique. La résonance magnétique nucléaire est particulièrement utile pour étudier la dynamique d'une molécule en solution sur une gamme de temps de corrélation très large. En particulière, la relaxation des $\operatorname{spins~}{ }^{15} \mathrm{~N}$ ou ${ }^{13} \mathrm{C}$ donne accès aux mouvements moléculaires avec les temps caractéristiques entre les dizaines de picosecondes et le temps de corrélation rotationelle de la molécule (autour de 10 ns pour une protéine monomérique de $20 \mathrm{kD}$ à $300 \mathrm{~K}$ ). Les vitesses de relaxation dépendent de la flexibilité de chaque site, et peuvent être caractérisé en termes d'amplitude et de temps caractéristique locale. La précision de ces paramètres et sa compréhension en termes de fonction exigent que la réorientation globale de la molécule soit correctement prise en compte. Ces méthodes expérimentales, qui seront présentées ici brièvement, font maintenant partie de la panoplie d'expériences appliquées dans l'étude de la relation structure-dynamique d'une protéine et ses partenaires. Néanmoins les mouvements plus lents, entre la nano et la milliseconde, sont plus difficiles à étudier, et il y a très peu d'information disponible sur la nature de la dynamique de la chaîne peptidique dans cette gamme de temps par RMN. Très récemment de nouvelles méthodologies ont été proposées, basées sur l'alignement préférentiel d'une protéine par rapport au champ magnétique, induit par dissolution de la molécule dans un cristal liquide très dilué. Dans ces conditions les changements conformationels sur des temps caractéristique plus lents (jusqu'au millisecondes) peuvent être étudiés. Nous présenterons cette technique, et quelques résultats, comparant la dynamique rapide (ps-ns), et plus lente le long de la chaîne peptidique de quatre protéines.
\end{abstract}

Table des matières

1 INTRODUCTION

2 COUPLAGES DIPOLAIRES RÉSIDUELS ET DYNAMIQUE : BASES

THÉORIQUES

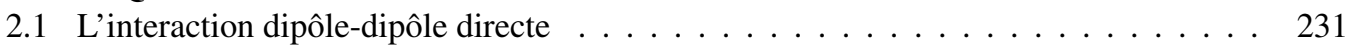

2.2 Couplages dipolaires résiduels en milieux orientants . . . . . . . . . . . . 233

3 UTILISATION DES CDR DANS L'ÉTUDE DYNAMIQUE 235

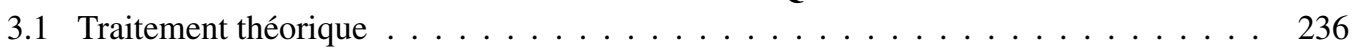

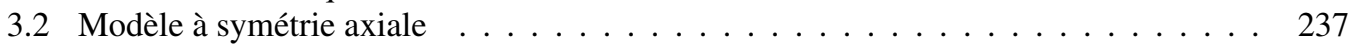

3.3 Modèle des fluctuations axiales Gaussiennes . . . . . . . . . . . . . . . 238

3.4 Modèle du saut à deux sites . . . . . . . . . . . . . . . . . . . 239

4 MÉTHODES NUMÉRIQUES

4.1 Le Tenseur d'alignement . . . . . . . . . . . . . . . . . . . . . . . . 240

4.2 Calcul du paramètre de dynamique . . . . . . . . . . . . . . 241

5 DÉTERMINATION DE LA MOBILITÉ LOCALE DANS LA PROTÉINE G 241 


\section{INTRODUCTION}

Visualiser la structure tridimensionnelle d'une protéine à l'échelle atomique est extrêmement utile pour comprendre sa fonction et son mécanisme. Cela n'est cependant pas suffisant pour une entière compréhension de son fonctionnement. Pour que l'étude soit complète, il est indispensable de s'intéresser à sa dynamique. En effet, hétéropolymères comportant un grand nombre de degrés de liberté, les protéines présentent une dynamique interne riche et complexe étroitement associée à leur réactivité à travers diverses catégories de mouvements: par exemple les fluctuations conformationnelles de surface permettant reconnaissance, interactions entre partenaires, entrée et la sortie de ligands ou de substrats, fluctuations structurales du site actif engendrant une distribution des vitesses de réaction, relaxation conformationnelle consécutive à la réaction

La Résonance Magnétique Nucléaire (RMN) occupe une position unique parmi les méthodes biophysiques de part sa grande sensibilité aux mouvements intramoléculaires des protéines. Les mouvements rapides, avec un temps caractéristique allant jusqu'au temps de corrélation de réorientation de la molécule, autour de $10 \mathrm{~ns}$ pour les protéines de taille moyenne en solution aqueuse à température ambiante, peuvent être étudiés directement par les mesures de relaxation de spin, une technique bien établie $[1,2]$. Ces méthodes expérimentales, combinées avec une analyse intuitivement simple en termes d'amplitude dynamique et de fréquences du mouvement, font partie de la panoplie classique des expériences généralement effectuées dans une étude de protéine par RMN [3,4]. Dans des échelles de temps plus importantes, l'échange conformationel entre des sites ayant des déplacements chimiques différents peut contribuer significativement au terme de relaxation transverse. Il est habituellement détecté par l'expérience de relaxation dispersion qui met en évidence l'équilibre conformationel sur une échelle de temps de l'ordre de la microseconde [5]. Ces échelles de temps sont particulièrement intéressantes car un grand nombre de processus biologiques fonctionnellement importants sont supposés s'y produire.

Récemment, l'utilisation de cristaux liquides dilués induisant un léger alignement des protéines par rapport au champ magnétique et introduisant, ainsi, une faible anisotropie dans l'espace orientationel de la molécule étudiée a suscité un vif intérêt $[6,7]$. Les couplages dipolaires résiduels alors mesurés donnent une information très précise de l'orientation des liaisons internucléaires relativement à un repère attaché à la molécule. Cette caractéristique rend ces couplages très puissants pour déterminer la structure de biomolécules [8-10]. Comme ces mesures proviennent de moyennes faites sur des échelles temps relativement longues (jusqu'à la milliseconde) ainsi que sur l'ensemble de la population,l'information dynamique qu'elles contiennent est complémentaire de celle obtenue par l'étude de la relaxation de spin et donc potentiellement très intéressante pour identifier des modèles spécifiques de mouvements. Dès les premières études sur l'alignement partiel des protéines, l'importance de ces particularités a été reconnue et a stimulé le développement de nombreuses approches méthodologiques permettant d'extraire des informations sur la dynamique à partir des couplages dipolaires [11-16].

Les récentes contributions se servant des couplages dipolaires résiduels pour étudier les moyennes conformationnelles ont utilisé des approches sans contrainte géométrique (model free) pour caractériser les propriétés de réorientation des vecteurs $\mathrm{N}^{\mathrm{N}} \mathrm{H}$ [17-20]. La direction et l'amplitude de l'échantillonnage angulaire du vecteur internucléaire sont décrits: soit purement à partir d'harmoniques sphériques moyennées de deuxième ordre $\left\langle\mathrm{Y}_{2 \mathrm{M}}\right\rangle$, qui sont ensuite déterminées explicitement; soit à partir de matrices décrivant aussi bien l'information structurale que dynamique, à déterminer aussi. Ces deux méthodologies ont été appliquées à partir des données expérimentales des couplages dipolaires résiduels $\mathrm{N}-{ }^{\mathrm{N}} \mathrm{H}$, mesurées dans 11 milieux d'alignement différents, de la protéine ubiquitine. La première de ces études détecte un degré de mobilité qui n'est pas visible à partir des études de relaxation, probablement dans la gamme de la nano à la milliseconde. Dans ce dernier cas, les auteurs ne présentent pas l'amplitude 
absolue des mouvements, mais seulement les paramètres d'ordre qui y sont relatifs. Abordant le problème différemment, une étude récente a démontré, en considérant, dans chaque peptide, plusieurs couplages dipolaires résiduels mesurés dans 5 milieux orientants, qu'une seule conformation, en accord avec tous les couplages, peut être déterminée. Une analyse de cette structure a conclu que l'amplitude des mouvements des vecteurs $\mathrm{N}^{\mathrm{N}} \mathrm{H}$ était moindre que celle déduite de l'approche décrite plus haut, l'origine de cette différence reste inconnue [21].

Un désavantage des approches développées par Brüschweiler et Griesinger, d'une part, et Tolman, d'autre part, est la nécessité, expérimentalement délicate, de trouver un grand nombre ( $>5)$ de milieux d'alignement différents. Pour parvenir à ce but, chaque milieu doit induire une orientation résiduelle de la molécule par rapport au champ magnétique significativement différente (voir ci-dessous, le tenseur d'alignement), qui, en pratique, suppose que l'interaction entre le soluté et le milieu soit différente dans chacun des cas. Il est donc intéressant de développer des méthodes alternatives, peut-être plus générales, pour détecter le moyennement conformationel à partir des couplages dipolaires résiduels.

Dans cette étude, nous allons développer une approche différente: au lieu d'essayer de déterminer explicitement les composantes des harmoniques sphériques, et donc la moyenne du couplage dipolaire, nous allons appliquer et tester des modèles géométriques simples pour décrire les mouvements locaux. Ainsi, nous allons réduire le nombre de paramètres indépendants nécessaires à caractériser les mouvements. Les mouvements de la chaîne peptidique sont certainement des processus complexes, ce qui implique qu' aucun modèle simple ne peut être universel. Néanmoins, nous avons choisi trois modèles intuitifs que nous développerons et dont nous testerons la pertinence devant les données expérimentales: le modèle à symétrie axiale, les fluctuations du plan peptidique autour d'un axe défini par les carbones alpha, un modèle de saut entre deux conformations autour de ce même axe et un modèle de réorientation à symétrie cylindrique du vecteur $\mathrm{N}^{-}{ }^{\mathrm{N}} \mathrm{H}$.

Ici, nous présentons une comparaison de ces différents modèles, suivie par l' application dans l'étude de la dynamique moléculaire de la protéine $\mathrm{G}$.

\section{COUPLAGES DIPOLAIRES RÉSIDUELS ET DYNAMIQUE : BASES THÉORIQUES}

\subsection{L'interaction dipôle-dipôle directe}

Chaque spin nucléaire est associé à un moment magnétique de spin et génère un champ magnétique dans l'espace environnant. Deux spins nucléaires se trouvant dans un même voisinage interagiront donc chacun avec l'autre: le premier « voyant » le champ magnétique créé par le second et inversement. L'interaction est mutuelle.

Contrairement aux couplages scalaires, l'interaction dipolaire ne nécessite pas de liaison électronique entre les deux atomes mis en jeu pour exister. Elle s'effectue sans intermédiaire entre les deux noyaux ; c'est pourquoi, elle s'appelle « interaction dipôle-dipôle directe ». Les couplages dipolaires peuvent donc être aussi bien intramoléculaires qu'intermoléculaires.

L'expression complète de l'interaction dipôle-dipôle entre les spins $I_{j}$ et $I_{k}$ est donnée par l'Hamiltonien de spin suivant [22, 23]:

$$
H_{j k}^{D D}=b_{j k}\left(3\left(\ddot{p}_{j} \cdot e_{j k}\right)\left(\ddot{p}_{k} \cdot e_{j k}\right)-\ddot{p}_{k} \cdot \ddot{p}_{k}\right)
$$

où $e_{j k}$ est le vecteur unitaire colinéaire à la droite joignant le noyau $j$ au noyau $k$.

L'amplitude dans l'interaction dipolaire est donnée par la constante de couplage dipolaire:

$$
b_{j k}=-\frac{\mu_{0}}{4 \pi} \frac{\gamma_{j} \gamma_{k} \hbar}{r_{j k}^{3}}
$$

où $\gamma_{j}$ et $\gamma_{k}$ sont les rapports gyromagnétiques des deux spins (en rad.s $\left.{ }^{-1}\right), \mu_{0}$ la permittivité du vide, $\hbar=h / 2 \pi$ avec $\mathrm{h}$ la constante de Planck et $r_{j k}$ la distance entre les deux spins (en m). 


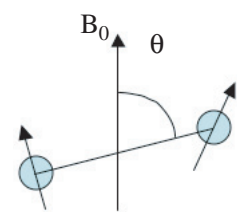

Figure 1. Représentation des deux spins, de l'orientation du champ magnétique B0 et de l'angle $\theta$.

L'amplitude de l'équation 2 est donnée en rad.s ${ }^{-1}$. Par exemple, pour la paire de spin ${ }^{15} \mathrm{~N}-{ }^{1} \mathrm{H}$, considérant la distance $r_{j k}=1.02 \AA$, l'amplitude de l'interaction est égale à $b_{j k} / 2 \pi=23 \mathrm{KHz}$.

Lorsque les spins sont placés dans un champ $\mathrm{B}_{0}$ intense, seule la composante des spins selon la direction du champ est significative dans l'Hamiltonien de l'interaction dipôle-dipôle direct. Ainsi, dans l'approximation des hauts champs, seule cette composante est prise en compte, les autres sont négligées. Si l'on suppose que le champ $\mathrm{B}_{0}$ est colinéaire à l'axe $\mathrm{z}$ alors l'Hamiltonien de l'équation 1 se réécrit de la façon suivante:

$$
H_{j k}^{D D}\left(\theta_{j k}\right)=2 d_{j k} \ddot{p}_{j z} \ddot{p}_{k z}
$$

où $\mathrm{d}_{j k}$ vaut:

$$
d_{j k}=\frac{1}{2} b_{j k}\left(3 \cos ^{2} \theta_{j k}-1\right)
$$

et $\theta_{j k}$ est l'angle que fait le vecteur internucléaire avec la direction du champ magnétique (Figure 1), c'est-à-dire:

$$
\cos \theta_{j k}=e_{j k} \cdot e_{z}
$$

Le cas particulier des liquides isotropes est important car les mesures en RMN des biomolécules s'effectuent couramment dans ce type de milieu. Dans un liquide isotrope, les molécules se réorientent uniformément et avec une vitesse suffisamment grande pour que seule la moyenne des couplages dipolaires intramoléculaires soit mesurable. L'isotropie de la réorientation rend cette moyenne nulle. Aucun couplage dipolaire intramoléculaire n'est donc mesurable dans ces milieux.

En effet, la probabilité $p(\Omega) d \Omega$ que la molécule s'oriente selon l'angle solide unitaire $\mathrm{d} \Omega=$ $\sin \theta_{j k} \mathrm{~d} \theta_{j k} \mathrm{~d} \varphi_{j k}\left(\theta_{j k}\right.$ et $\varphi_{j k}$ étant les angles polaires du vecteur internucléaire par rapport au champ $\left.\mathrm{B}_{0}\right)$ est donnée par:

$$
p\left(\theta_{j k}, \varphi_{j k}\right) d \Omega=\frac{1}{4 \pi} \sin \theta_{j k} d \theta_{j k} d \varphi_{j k}
$$

Donc:

$$
\left\langle 3 \cos ^{2} \theta_{j k}-1\right\rangle=\frac{1}{4 \pi} \int_{0}^{2 \pi} d \varphi_{j k} \int_{0}^{\pi} d \theta_{j k}\left(3 \cos ^{2} \theta_{j k}-1\right) \sin \theta_{j k}=0
$$

D'où:

$$
\left\langle H_{j k}^{D D}\left(\theta_{j k}\right)\right\rangle=0
$$

De façon analogue, les interactions dipôle-dipôle intermoléculaires à courte distance sont moyennées à zéro du fait du mouvement de translation des molécules. Les interactions dipôle-dipôle à plus grande distance ne sont pas moyennées, mais sont trop faibles pour être mesurées. Ainsi, dans un liquide isotrope, en solution aqueuse par exemple, les couplages dipolaires ne sont pas mesurables. Cela à l'avantage de rendre les raies de résonance d'un spectre de RMN en solution plus fines et donc plus lisibles. Cependant, il n'est aussi pas possible d'avoir accès aux précieuses informations de l'interaction dipôle-dipôle direct. A l'opposé, en RMN du solide, les couplages sont si importants qu'ils rendent rapidement illisible le 


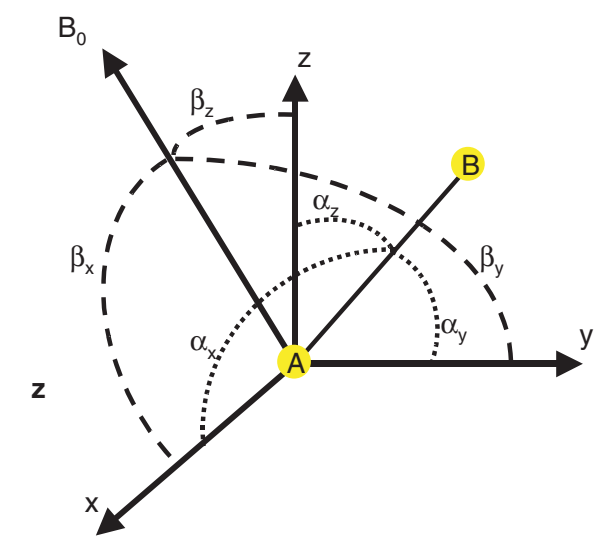

Figure 2. Orientation du champ $\mathrm{B}_{0}$ et du vecteur internucléaire dans le repère lié à la molécule.

spectre d'une protéine. Entre ces deux extrêmes, un compromis a été trouvé dans les liquides anisotropes. Ceux-ci grâce à leurs caractéristiques particulières ont permis l'exploitation des couplages dipolaires directs en RMN en solution.

\subsection{Couplages dipolaires résiduels en milieux orientants}

Une des plus récentes avancées dans le monde de la RMN haute résolution est venue de l'introduction des milieux orientants pour le calcul de structure et de dynamique. Dans de tels milieux, les protéines sont partiellement orientées. Leur espace orientationel devient alors suffisamment anisotrope pour permettre la mesure précise de couplages dipolaires résiduels (CDR) [8-10]. L'anisotropie induite par ces milieux reste suffisamment faible pour ne pas perturber le comportement des protéines et préserver au spectre RMN sa lisibilité.

Nous supposerons, dans un premier temps, la molécule comme une entité rigide. Les distances ainsi que les orientations en son sein sont supposées constantes et seule son orientation par rapport au champ magnétique $\mathrm{B}_{0}$ varie.

La valeur du couplage dipolaire entre deux spins $I_{j}$ et $I_{k}$ de la molécule s'écrit alors:

$$
D_{j k}=b_{j k}\left\langle\frac{3 \cos ^{2} \theta_{j k}-1}{2}\right\rangle
$$

où les crochets représentent la moyenne temporelle ou d'ensemble (le phénomène est ergodique) de la réorientation de la molécule.

L'orientation de chaque vecteur internucléaire $e_{j k}$, dans n'importe quel repère lié à la molécule, peut être défini par les angles $\alpha_{x}, \alpha_{y}$ et $\alpha_{z}$ que fait le vecteur avec les axes du système de coordonnée. De la même manière, les angles $\beta_{x}, \beta_{y}$ et $\beta_{z}$ définissent l'orientation instantanée du champ $B_{0}$ dans le repère lié à la molécule (Figure 2).

D'après l'équation $3, \cos \theta$ peut être réécrit sous la forme suivante:

$$
\cos \theta=\left(\begin{array}{c}
\cos \alpha_{x} \\
\cos \alpha_{y} \\
\cos \alpha_{z}
\end{array}\right) \cdot\left(\begin{array}{c}
\cos \beta_{x} \\
\cos \beta_{y} \\
\cos \beta_{z}
\end{array}\right)=\cos \alpha_{x} \cos \beta_{x}+\cos \alpha_{y} \cos \beta_{y}+\cos \alpha_{z} \cos \beta_{z}
$$

Et alors:

$$
\left\langle\frac{3 \cos ^{2} \theta_{j k}-1}{2}\right\rangle=\frac{3}{2}\left\langle\left(\cos \alpha_{x} \cos \beta_{x}+\cos \alpha_{y} \cos \beta_{y}+\cos \alpha_{z} \cos \beta_{z}\right)^{2}\right\rangle-\frac{1}{2}
$$


Avec $c_{i}=\cos \alpha_{i}$ et $C_{i}=\cos \beta_{i}$, on obtient:

$$
\begin{aligned}
\left\langle\frac{3 \cos ^{2} \theta_{j k}-1}{2}\right\rangle= & \frac{3}{2}\left[\left\langle C_{x}^{2}\right\rangle c_{x}^{2}+\left\langle C_{y}^{2}\right\rangle c_{y}^{2}+\left\langle C_{z}^{2}\right\rangle c_{z}^{2}+2\left\langle C_{x} C_{y}\right\rangle c_{x} c_{y}\right. \\
& \left.+2\left\langle C_{x} C_{z}\right\rangle c_{x} c_{z}+2\left\langle C_{y} C_{z}\right\rangle c_{y} c_{z}\right]-\frac{1}{2}
\end{aligned}
$$

Soit S la matrice définie par: $S_{i j}=\frac{3}{2}\left\langle C_{i} C_{j}\right\rangle-\frac{1}{2} \delta_{i j}$ où $\delta_{i j}$ est la fonction de Kronecker. Alors (12b) s'écrit aussi:

$$
\left\langle\frac{3 \cos ^{2} \theta_{j k}-1}{2}\right\rangle=\sum_{i, j=\{x, y, z\}} S_{i j} \cos \alpha_{i} \cos \alpha_{j}
$$

La matrice $3 \times 3 \mathrm{~S}$ est communément appelée la matrice de Saupe ou simplement la matrice d'ordre [24]. Comme $\left\langle C_{x}^{2}\right\rangle+\left\langle C_{y}^{2}\right\rangle+\left\langle C_{z}^{2}\right\rangle=1$, la matrice $S$ est de trace nulle et, comme $\left\langle C_{x} C_{y}\right\rangle=\left\langle C_{y} C_{x}\right\rangle$, S est aussi symétrique. Elle ne contient donc que cinq termes indépendants. Si la structure de la molécule est connue, c'est-à-dire que les $\cos \alpha_{i}$ sont connus, la valeur des cinq termes indépendants de la matrice peut généralement être déterminée à partir de la valeur du couplage dipolaire d'au moins cinq vecteurs internucléaires différents. Dans l'étude d'une macromolécule biologique, beaucoup plus de couplages sont généralement mesurés et $\mathrm{S}$ est surdéterminée.

La matrice $\mathrm{S}$ est réelle et symétrique, il est donc toujours possible de trouver un repère lié à la molécule dans lequel S est diagonale. Dans la suite, on se placera toujours dans le repère qui diagonalise cette matrice, repère dont on caractérisera la position dans le repère de la molécule par les angles d'Euler $\left(\xi_{1}, \xi_{2}, \xi_{3}\right)$. L'équation (12b) devient alors:

$$
D_{j k}\left(\alpha_{x}, \alpha_{y}, \alpha_{z}\right)=b_{i j}\left\{\frac{3}{2}\left[\left\langle C_{x}^{2}\right\rangle c_{x}^{2}+\left\langle C_{y}^{2}\right\rangle c_{y}^{2}+\left\langle C_{z}^{2}\right\rangle c_{z}^{2}\right]-\frac{1}{2}\right\}
$$

où $\left\langle C_{i}^{2}\right\rangle$ correspond à la probabilité de trouver l'axe $i$ colinéaire au champ magnétique.

Seule la différence relative entre les valeurs $\left\langle C_{i}^{2}\right\rangle$ contribue au couplage dipolaire résiduel.

En écrivant $\left\langle C_{i}^{2}\right\rangle=\frac{1}{3}+A_{i i}$ et en coordonnées polaires $\theta=\alpha_{z} ; c_{z}=\cos \theta ; c_{x}=\sin \theta \cos \phi ; c_{y}=$ $\sin \theta \sin \phi$; l'équation (13a) peut être réécrite:

$$
D_{j k}(\theta, \phi)=\frac{3}{2} b_{i j}\left[A_{z z} \cos ^{2} \theta+A_{x x} \sin ^{2} \theta \cos ^{2} \phi+A_{y y} \sin ^{2} \theta \sin ^{2} \phi\right]
$$

On suppose que $\left|A_{x x}\right|<\left|A_{y y}\right|<\left|A_{z z}\right|$. Il est toujours possible de se ramener à ce cas, l'hypothèse n'enlève donc rien à la généralité du raisonnement.

En utilisant le fait que: $A_{x x}+A_{y y}=-A_{z z} ; 2 \cos ^{2} \phi=1+\cos 2 \phi ; 2 \sin ^{2} \phi=1-\cos 2 \phi$; la formule se réécrit:

$$
D_{j k}(\theta, \phi)=\frac{3}{2} b_{i j}\left[A_{z z}\left(\frac{3 \cos ^{2} \theta-1}{2}\right)+\left(A_{x x}-A_{y y}\right) \frac{1}{2} \sin ^{2} \theta \cos 2 \phi\right]
$$

Si on définit la composante axiale $A_{a}$ et rhombique $A_{r}$ du tenseur A comme:

$$
A_{a}=\frac{3}{2} A_{z z} \text { et } A_{r}=A_{x x}-A_{y y}
$$

l'équation devient:

$$
D_{j k}(\theta, \phi)=b_{i j}\left[A_{a}\left(\frac{3 \cos ^{2} \theta-1}{2}\right)+\frac{3}{4} A_{r} \sin ^{2} \theta \cos 2 \phi\right]
$$

Une des caractéristiques propres aux couplages dipolaires résiduels est leur dégénérescence (Figure 3), c'est-à-dire qu'une valeur donnée ne correspond pas à une orientation unique du vecteur internucléaire étudié. On lève ces dégénérescences en introduisant une cohérence entre les différentes données en 

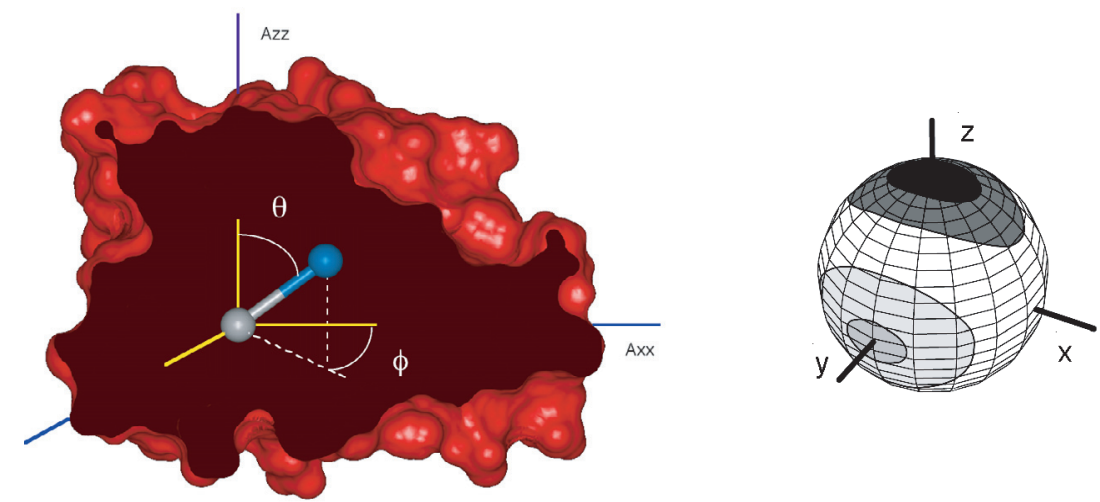

Figure 3. A gauche ${ }^{\circ}$ : Coordonnés d'un vecteur internucléaire dans le repère principal du tenseur d'alignement. A droite $^{\circ}$ : Isocontours des CDR par rapport à un tenseur $\mathrm{d}^{\prime}$ alignement ${ }^{\circ}$; la valeur des CDR va croissant du gris au noir en passant par le blanc.

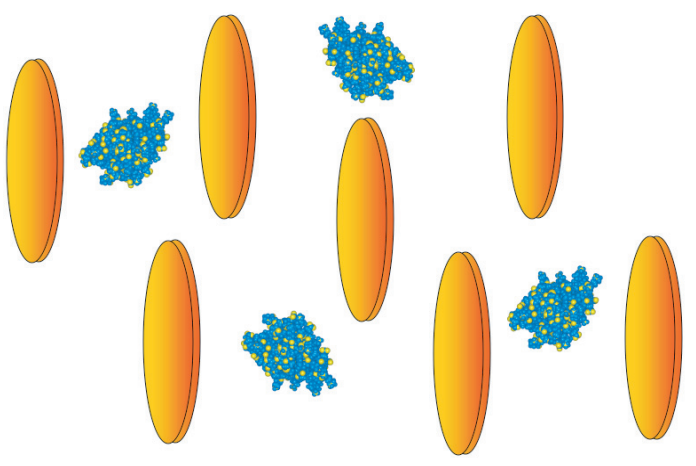

Figure 4. Milieu orientant.

considérant des motifs structuraux particuliers ou en utilisant des mesures relativement à deux tenseurs dont les orientations et les valeurs propres diffèrent [25].

Maintenant il existe beaucoup de milieux orientants [26] qu' on a coutume de classer selon leur forme, leur mode d'interaction et leur comportement physique [27]. (Figure 4). Pour qu'il soit possible d'étudier une macromolécule dans ces milieux, l'ordre imposé à la molécule doit être très petit, typiquement moins de 0,002. L'ordre des particules du cristal liquide est, quant à lui, grand; il se situe entre 0,5 et 0,85 . Il est clair que les interactions des macromolécules avec le cristal liquide doivent être faibles. Par ailleurs, les milieux doivent être aqueux pour que les protéines soient dans un environnement proche de leur milieu naturel. Ces conditions sont satisfaites en utilisant des solutions aqueuses de cristaux liquides fortement diluées ou les milieux avec les cavités anisotropes.

\section{UTILISATION DES CDR DANS L'ÉTUDE DYNAMIQUE}

Nous avons supposé, pour le moment, que les macromolécules étudiées étaient rigides. Pourtant, comme nous l'avons dit précédemment, il est maintenant bien établi que les macromolécules en solution possèdent une dynamique interne qui est sans doute très importante sur le plan biologique.

Les CDR constituent une autre voie d'étude de la dynamique alternative a la relaxation. Bien que les phénomènes physiques mesurés dans les deux cas soient différents, l'objet d'étude, lui, est identique et concerne la réorientation des mêmes vecteurs. Ainsi, les spécificités de chacune des méthodes sont 
autant de visions différentes d'un même mouvement: les deux méthodes sont donc complémentaires [11]. L'utilisation des CDR permet d'étudier les mouvements qui s'échelonnent sur une gamme de temps plus importante (jusqu'à la milliseconde). Malgré ce potentiel important pour étudier les mouvements plus lents, très peu d'études utilisant des modèles géométriques de mobilité locale dans les protéines ont été décrits dans la littérature. C'est à ce dernier point que s'intéressera la présente partie. Trois modèles de dynamique moléculaire seront présentés ainsi que la formulation analytique du CDR qui leur est associé.

\subsection{Traitement théorique}

En présence de dynamique intramoléculaire, les couplages dipolaires mesurés correspondent à une moyenne conformationnelle. Les crochets représentants la moyenne angulaire, la valeur des couplages dipolaires mesurés est donnée par:

$$
\left\langle D_{j k}(\theta, \phi)\right\rangle=b_{i j}\left[A_{a}\left\langle\frac{3 \cos ^{2} \theta-1}{2}\right\rangle+\frac{3}{4} A_{r}\left\langle\sin ^{2} \theta \cos 2 \phi\right\rangle\right]
$$

L'équation 14 suppose que les mouvements intramoléculaires n'interfèrent pas avec le processus d'alignement, c'est-à-dire que le processus d'alignement n'est pas affecté de façon significative par les mouvements internes.

On peut aussi l'exprimer à l'aide d'harmoniques sphériques moyennées [28]:

$$
\left\langle D_{j k}(\theta, \phi)\right\rangle=\sqrt{\frac{16 \pi}{5}} b_{i j}\left[A_{a}\left\langle Y_{20}(\theta, \phi)\right\rangle+\sqrt{\frac{3}{8}} A_{r}\left(\left\langle Y_{22}(\theta, \phi)\right\rangle+\left\langle Y_{2-2}(\theta, \phi)\right\rangle\right)\right]
$$

Cette expression a l'avantage d'être facilement transposable d'un repère à un autre en utilisant les propriétés relatives aux rotations d'Euler des harmoniques sphériques. Pour une rotation de repère d'angle $(\alpha, \beta, \gamma)$, notée $\mathrm{R}(\alpha, \beta, \gamma)$, on a:

$$
R(\alpha, \beta, \gamma) Y_{2, M}(\theta, \phi)=\sum_{M^{\prime}=-2}^{+2} e^{-i \alpha M^{\prime}} d_{M^{\prime} M}^{(2)}(\beta) e^{-i \gamma M} Y_{2, M^{\prime}}(\theta, \phi)
$$

où $d_{M^{\prime} M}^{(2)}$ est la matrice de Wigner d'ordre 2.

En suivant la précédente logique, on écrit, d'abord, la moyenne des harmoniques sphériques dans un repère lié au plan peptidique pour, ensuite, la transposer dans le repère lié au tenseur d'alignement en utilisant la rotation d'Euler appropriée au plan peptidique considéré (voir Figure 5). On a alors:

$$
\left\langle D_{j k}(\theta, \phi)\right\rangle=\sqrt{\frac{16 \pi}{5}} b_{j k}\left[\begin{array}{l}
A_{a}\left(\sum_{M^{\prime}=-2}^{+2} e^{-i \alpha M^{\prime}} d_{M^{\prime} 0}^{(2)}(\beta)\left\langle Y_{2, M^{\prime}}\right\rangle\right)+ \\
\sqrt{\frac{3}{8}} A_{r}\left(\begin{array}{c}
\sum_{M^{\prime}=-2}^{+2} e^{-i \alpha M^{\prime}} d_{M^{\prime} 2}^{(2)}(\beta) e^{-i 2 \gamma}\left\langle Y_{2, M^{\prime}}\right\rangle+ \\
\sum_{M^{\prime}=-2}^{+2} e^{-i \alpha \cdot M^{\prime}} d_{M^{\prime}-2}^{(2)}(\beta) e^{+i 2 \gamma}\left\langle Y_{2, M^{\prime}}\right\rangle
\end{array}\right)
\end{array}\right]
$$

Dans ce cas, la rotation $(\alpha, \beta, \gamma)$ décrit le passage du repère principal du tenseur d'alignement au repère local lié au plan peptidique considéré. Il existe 16 conventions différentes pour définir les angles d'Euler. Nous utiliserons toujours la convention Z-Y-Z. Ceci permet de traiter analytiquement la dynamique de toute la molécule de façon identique.

L'équation 17 est linéaire par rapport à la moyenne dynamique des cinq harmoniques. Si les couplages $\left\langle D_{j k}\right\rangle$ d'une même paire de spin étaient mesurés dans cinq (ou plus) tenseurs d'alignement connus $\left\{A_{a}\right.$, $\left.A_{r}, \xi_{1}, \xi_{2}, \xi_{3}\right\}$, les cinq valeurs $\left\langle Y_{2, M^{\prime}}\right\rangle$ pourraient être déterminées en résolvant le système linéaire constitué à partir de l'équation 17 , sans recours aux modèles géométriques [17, 19]. Cependant, il est difficile d'obtenir la mesure d'un couplage dans plus de 5 milieux différents et le cas de l'ubiquitine reste exceptionnel. 


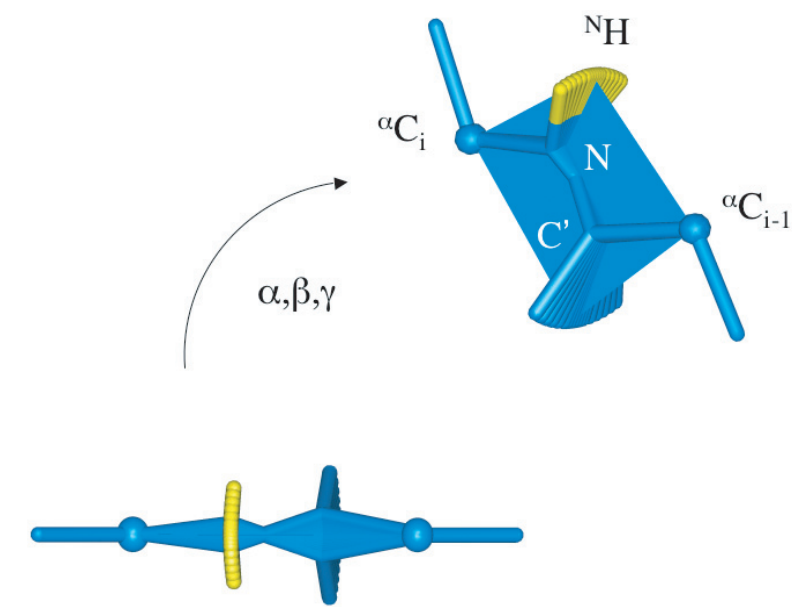

Figure 5. Illustration du mouvement FAG et du passage du repère peptidique au repère principal de la molécule par la rotation d'angle $(\alpha, \beta, \gamma)$. Le vecteur $\mathrm{N}^{\mathrm{N}} \mathrm{H}$ est en jaune.

Un des buts de notre travail est de réduire le nombre de paramètres nécessaires à la description du mouvement grâce à des modèles géométriques spécifiques. Il est aujourd'hui peu probable qu'un modèle unique de dynamique puisse suffire à la description du mouvement de la chaîne polypeptidique d'une protéine. Ici nous allons développer trois différents qui représentent les modes du mouvement les plus intuitifs.

Dans le développement des trois modèles de dynamique moléculaire suivant, seule la paire nucléaire $\mathrm{N}-{ }^{\mathrm{N}} \mathrm{H}$ de chaque peptide sera traitée. L'intérêt particulier que suscite ce couplage se justifie par sa facilité de mesure et sa grande dynamique, la plus importante de tous les couplages du plan peptidique [29].

\subsection{Modèle à symétrie axiale}

Le modèle à symétrie axiale a été introduit, d'abord, pour son côté intuitif et sa grande simplicité. Il est censé représenter le mouvement d'une paire atomique en l'absence d'anisotropie. Il se caractérise par une distribution à symétrie axiale du vecteur internucléaire autour d'une position moyenne. Les angles d'Euler $(\alpha, \beta, \gamma)$ sont choisis de telle sorte que l'axe $\mathrm{z}$ du repère lié au plan peptidique soit le long de la position moyenne du vecteur internucléaire considéré. Analytiquement cela se traduit par:

$$
\left\langle e^{ \pm i \phi}\right\rangle=\left\langle e^{ \pm 2 i \phi}\right\rangle=0
$$

D'où:

$$
\left\langle Y_{2, \pm 2}(\theta, \phi)\right\rangle=\left\langle Y_{2, \pm 1}(\theta, \phi)\right\rangle=0
$$

L'équation 17 se simplifie alors:

$$
\left\langle D_{j k}(\theta, \phi)\right\rangle=\sqrt{\frac{16 \pi}{5}} b_{i j}\left\langle Y_{2,0}(\theta, \phi)\right\rangle\left[A_{a} d_{00}^{(2)}(\beta)+\sqrt{\frac{3}{8}} A_{r}\left(d_{02}^{(2)}(\beta) e^{-i 2 \gamma}+d_{0-2}^{(2)}(\beta) e^{+i 2 \gamma}\right)\right]
$$

En explicitant les termes de la matrice de Wigner, nous obtenons:

$$
\left\langle D_{j k}(\theta, \phi)\right\rangle=b_{i j}\left\langle\frac{3 \cos ^{2} \theta-1}{2}\right\rangle\left[A_{a}\left(\frac{3 \cos ^{2} \beta-1}{2}\right)+\frac{3}{4} A_{r} \sin ^{2} \beta \cos 2 \gamma\right]
$$


où $\beta=-\theta_{\text {statique }}$ et $\gamma=-\phi_{\text {statique }}$ avec $\left(\theta_{\text {statique }}, \phi_{\text {statique }}\right)$ l'orientation de l'axe de symétrie du mouvement. Réécrit autrement, cela devient:

$$
\left\langle D_{j k}(\theta, \phi)\right\rangle=S . D_{j k \text { statique }}\left(\theta_{\text {statique }}, \phi_{\text {statique }}\right)
$$

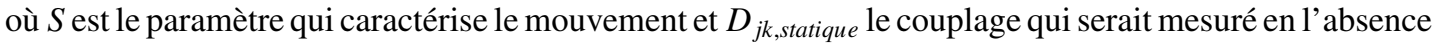
de dynamique.

L'effet de la dynamique dû aux mouvements de symétrie axiale ne dépend pas de l'orientation du vecteur internucléaire, mais uniquement de l'amplitude du mouvement. Le paramètre, noté $S$, est à rapprocher du paramètre d'ordre issu des études de relaxation. En effet, ce dernier s'écrit de façon générale:

$$
S^{2}=\frac{4 \pi}{5} \sum_{M=-2}^{+2}\left\langle Y_{2, M}(\theta, \phi)\right\rangle\left\langle Y_{2, M}^{*}(\theta, \phi)\right\rangle
$$

qui se simplifie, dans notre cas, et donne:

$$
S^{2}=\frac{4 \pi}{5}\left\langle Y_{2,0}(\theta, \phi)\right\rangle^{2}=\left\langle\frac{3 \cos ^{2} \theta-1}{2}\right\rangle^{2}
$$

\subsection{Modèle des fluctuations axiales Gaussiennes}

Même si la dynamique de la chaîne principale d'une protéine est complexe, des simulations de dynamique moléculaire, à partir des peptides et de l'ubiquitine, ont montré que des mouvements anisotropes, de la forme de Fluctuations Axiales Gaussiennes (FAG) autour d'une position moyenne, étaient particulièrement aptes à représenter le mouvement fondamental de la chaîne polypeptidique [32]. Brüschweiler et son équipe ont montré que le mouvement d'un peptide (supposé plan et rigide) pouvait être décrit par un mouvement FAG tridimensionnel défini par trois rotations aléatoires et indépendantes autour de trois axes orthogonaux, l'axe principal étant le long de l'axe $C_{i-1}^{\alpha}-C_{i}^{\alpha}[30-34]$. Une simplification du modèle FAG-3D peut être raisonnablement faite en ne prenant en compte que cette composante et en négligeant les autres. En effet, il a été montré que la convolution d'un mouvement FAG-1D avec un mouvement à symétrie axiale équivalait, de manière presque parfaite, à un mouvement FAG-3D [29].

Par ailleurs, pour décrire l'effet de moyennage de ce mouvement sur les CDR du couple $\mathrm{N}-{ }^{\mathrm{N}} \mathrm{H}$, nous supposerons, pour la suite, que le vecteur $\mathrm{N}^{-}{ }^{\mathrm{N}} \mathrm{H}$ est perpendiculaire au vecteur défini par le couple $\mathrm{C}_{\mathrm{i}-1}^{\alpha}-\mathrm{C}_{\mathrm{i}}^{\alpha}$. Il a été montré que cette approximation reproduit de manière satisfaisante les amplitudes simulées à partir du modèle FAG-1D. On l'appellera modèle ortho-FAG [29].

Les angles d'Euler utilisés dans l'équation 17 nous place dans la configuration suivante: l'axe z est le long de $\mathrm{N}^{\mathrm{N}} \mathrm{H}$ et l'axe y est dans le plan peptidique, approximativement le long de $\mathrm{C}_{\mathrm{i}-1}^{\alpha}-\mathrm{C}_{\mathrm{i}}^{\alpha}$ (Figure 5).

La formule 17, en présence de fluctuations axiales gaussiennes par rapport à l'axe $y$ du repère peptidique $(\phi=0)$, en utilisant:

$$
\langle\cos (m \theta)\rangle=\int_{-\infty}^{+\infty} p(\theta) \cos (m \theta) d \theta=\exp \left(\frac{-m^{2} \sigma^{2}}{2}\right)
$$

où:

$$
p(\theta)=\frac{1}{\sqrt{2 \pi \sigma^{2}}} \exp \left(\frac{-\theta^{2}}{2 \sigma^{2}}\right)
$$

devient:

$$
\left\langle D_{N H}\right\rangle=b_{i j}\left[\begin{array}{l}
\frac{A_{a}}{4}\left\{s_{1}\left(3 \cos ^{2} \beta-1\right)+3 s_{2} \sin ^{2} \beta \cos 2 \alpha\right\}+ \\
\frac{3}{8} A_{r}\left\{s_{1} \sin ^{2} \beta \cos 2 \gamma+2 s_{2}\left(\cos ^{4} \frac{\beta}{2} \cos 2 \delta_{1}+\sin ^{4} \frac{\beta}{2} \cos 2 \delta_{2}\right)\right\}
\end{array}\right]
$$


avec:

$$
\delta_{1}=\alpha+\gamma ; \quad \delta_{2}=\alpha-\gamma ; \quad s_{1}=1+3 e^{-2 \sigma^{2}} ; \quad s_{2}=1-e^{-2 \sigma^{2}}
$$

L'expression analytique du paramètre d'ordre $S$ relatif à un mouvement ortho-FAG d'amplitude $\sigma$ est la suivante [30]:

$$
S^{2}=1-\frac{3}{4}\left(1-e^{-4 \sigma^{2}}\right)
$$

\subsection{Modèle du saut à deux sites}

Le modèle du saut à deux sites décrit les sauts de conformations entre deux sites, chacune des conformations étant équiprobable. De la même manière que pour le modèle FAG-3D, il est raisonnable d'approximer le saut à deux sites d'un peptide comme une rotation par rapport à l'axe $C_{i-1}^{\alpha}-C_{i}^{\alpha} d$ 'angle $2 \theta$ centrée sur la position d'équilibre de $\mathrm{N}^{\mathrm{N}} \mathrm{H}$. On supposera, comme pour le FAG, que le vecteur $\mathrm{N}^{\mathrm{N}} \mathrm{H}$ est perpendiculaire à l'axe $\mathrm{C}_{\mathrm{i}-1}^{\alpha}-\mathrm{C}_{\mathrm{i}}^{\alpha}$.

Dans le repère lié au peptide i considéré, la loi de probabilité angulaire s'écrit:

$$
p(x)=\frac{1}{2}(\delta(x+\theta)+\delta(x-\theta))
$$

Les harmoniques sphériques sont alors moyennées de la façon suivante:

$$
\begin{gathered}
\left\langle Y_{2-2}\right\rangle=\frac{1}{4} \sqrt{\frac{15}{2 \pi}} \sin ^{2} \theta e^{-2 i \varphi} \\
\left\langle Y_{2-1}\right\rangle=0 \\
\left\langle Y_{20}\right\rangle=\frac{1}{4} \sqrt{\frac{5}{\pi}}\left(3 \cos ^{2} \theta-1\right) \\
\left\langle Y_{21}\right\rangle=0 \\
\left\langle Y_{22}\right\rangle=-\frac{1}{4} \sqrt{\frac{15}{2 \pi}} \sin ^{2} \theta e^{+2 i \varphi}
\end{gathered}
$$

où $\theta$ représente la demi-amplitude du saut.

L'expression du couplage dipolaire dans le repère principal du tenseur d'alignement s'obtient en réécrivant l'équation 17 et en explicitant les harmoniques sphériques moyennées:

$$
\left\langle D_{N H}\right\rangle=b_{i j}\left[\begin{array}{l}
\frac{A_{a}}{4}\left\{s_{1}^{\prime}\left(3 \cos ^{2} \beta-1\right)+3 s_{2}^{\prime} \sin ^{2} \beta \cos 2 \alpha\right\}+ \\
\frac{3}{8} A_{r}\left\{s_{1}^{\prime} \sin ^{2} \beta \cos 2 \gamma+2 s_{2}^{\prime}\left(\cos ^{4} \frac{\beta}{2} \cos 2 \delta_{1}+\sin ^{4} \frac{\beta}{2} \cos 2 \delta_{2}\right)\right\}
\end{array}\right]
$$

avec:

$$
\delta_{1}=\alpha+\gamma ; \delta_{2}=\alpha-\gamma ; s_{1}^{\prime}=2\left(3 \cos ^{2} \theta-1\right) ; s_{2}^{\prime}=2 \sin ^{2} \theta
$$

L'expression analytique du paramètre d'ordre $S$ relatif à un saut à deux sites d'amplitude $2 \theta$ est la suivante:

$$
S^{2}=\frac{3 \cos ^{2} 2 \theta+1}{4}
$$

La formule liée au saut à deux sites est remarquablement proche de celle du modèle de l'ortho-FAG. Une étude plus poussée montre qu'elle se comporte aussi de manière très semblable. Ainsi, jusqu'à une 


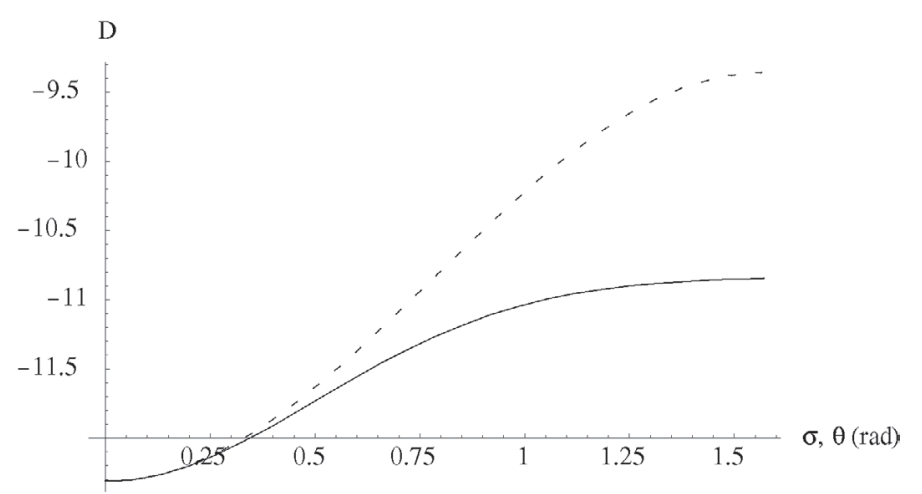

Figure 6. Comparaison entre le modèle FAG (trait plein) et saut à deux sites (pointillés), D étant la valeur du couplage (pas à 1'échelle) $\left(\alpha=\beta=\gamma=60^{\circ}, A_{a}=20.10^{-4}, A_{r}=13.10^{-4}\right)$.

amplitude de $43.5^{\circ}$ (0,76 radians), il est impossible de distinguer le modèle du saut à deux sites du modèle FAG, puisque, jusqu'à cet angle, les deux modèles balayent les mêmes valeurs de CDR. Cette ressemblance entre les modèles FAG et du saut à deux sites est illustrée dans la Figure 6.

\section{MÉTHODES NUMÉRIQUES}

\subsection{Le Tenseur d'alignement}

Comme on l'a vu précédemment, le tenseur d'alignement caractérise l'orientation des macromolécules en solution. C'est une matrice réelle d'ordre 3, de rang 2, symétrique et de trace nulle. Elle ne possède donc que cinq paramètres indépendants et est toujours diagonalisable dans une base orthonormée. On a l'habitude de le définir à l'aide des trois angles d'Euler $\left(\xi_{1}, \xi_{2}, \xi_{3}\right)$ caractérisant son repère principal (dans lequel il est diagonal) par rapport au repère absolu (dans lequel sont données les coordonnées des atomes de la protéine); et aussi de sa composante axiale $A_{a}$ et rhombique $A_{r}$ défini par les équations 13.

Le calcul du tenseur d'alignement a été effectué à partir de la structure de la protéine et des couplages dipolaires disponibles avec le logiciel MODULE [35]. La structure apporte les coordonnées des vecteurs internucléaires associés aux valeurs des CDR. Le tenseur possédant cinq paramètres indépendants, cinq couplages, au moins, et leur vecteur internucléaire associé doivent être connus pour déterminer complètement le tenseur. Dans la pratique, un maximum de couplages est utilisé. Cela permet de minimiser les erreurs dues au bruit contenu dans la valeur des couplages mesurés et dans la structure utilisée.

L'algorithme de détermination effectue une régression par la méthode des moindres carrés. Plus particulièrement, il minimise, en jouant sur les paramètres de la matrice, la fonction $\chi^{2}$ suivante:

$$
\chi^{2}\left(\xi_{1}, \xi_{2}, \xi_{3}, A_{a}, A_{r}\right)=\sum_{i}\left(\frac{D_{i}^{\text {exp }}-D_{i}^{\text {calc }}}{\sigma_{i}}\right)^{2}
$$

où $D_{i}^{\text {exp }}$ est la valeur expérimentale du couplage dipolaire $i, D_{i}^{\text {calc }}$ est la valeur du couplage dipolaire $i$ calculé via l'équation $13 \mathrm{~d}$ et $\sigma_{i}$ représente l'erreur sur la mesure du couplage dipolaire $i$. Ainsi, plus l'erreur sur un couplage sera grande moins celui-ci aura de poids dans le $\chi^{2}$. La méthode de minimisation s'appuie sur deux algorithmes: un algorithme de recuit simulé et l'algorithme de Levenberg-Marquardt. Les deux algorithmes travaillent de manière complémentaire: l'algorithme de Levenberg-Marquardt est particulièrement bien adapté à la recherche d'un minimum de l'espace paramétrique et le recuit simulé permet d'identifier les différents minima de ce même espace. L'association des deux est donc particulièrement efficace pour déterminer le minimum de l'espace paramétrique défini par le $\chi^{2}$. 
Nous avons choisi pour définir le $\chi^{2}$ d'utiliser le couplage dipolaire statique du résidu $i$. Ce choix se justifie par le fait que nous ne connaissons pas, à priori, la dynamique liée à ce résidu. Pourtant, un des points essentiels de notre étude est de montrer que la dynamique influe de manière non négligeable sur la valeur des CDR. Il est donc important de mentionner que les paramètres du tenseur d'alignement déterminés par la méthode «classique », sans prendre en compte les effets de la dynamique locale, seront influencés par un effet de moyennage complexe de tous ces mouvements et que les amplitudes locales extraites grâce à ce tenseur seront aussi affectées. Nous présentons deux approches pour résoudre ce problème. La première est d'utiliser les couplages $\mathrm{N}^{-}{ }^{\mathrm{N}} \mathrm{H}$ et de remplacer l'expression du couplage statique par la formule 27 du FAG-1D en optimisant un paramètre supplémentaire, $\langle\sigma\rangle$ représentant une amplitude moyenne de tous les sites considérés. Il a été montré que cela améliore significativement la justesse des calculs. Cette amélioration constitue d'ailleurs une preuve supplémentaire que le mouvement FAG est bien une composante de la dynamique de la protéine [29]. La seconde, valable si l'on considère un mouvement FAG, consiste à prendre pour calculer le $\chi^{2}$ uniquement les couplages des paires nucléaires qui subissent le moins de dynamique, c'est-à-dire les couples $C_{i-1}^{\alpha}-C_{i}^{\alpha}$ de chaque peptide [29].

\subsection{Calcul du paramètre de dynamique}

Une fois obtenue l'expression des tenseurs d'alignement et les angles d'Euler $(\alpha, \beta, \gamma)$, tous les paramètres des différents modèles de dynamique sont connus excepté le paramètre de dynamique. Il est alors relativement facile de le déterminer en l'ajustant par rapport aux données expérimentales. Cependant, ce paramètre peut être mal défini s'il est calculé à partir d'un unique tenseur d'alignement. On peut démontrer que certaines orientations du plan peptidique rendent l'effet d'un mouvement FAG presque ou complètement inobservable (par exemple, si le vecteur est placé sur un isocontour, voir Figure 3), c'est-à-dire qu'avec ou sans FAG, dans ces directions, la valeur du CDR calculé est la même [29]. Il est donc nécessaire, pour une analyse robuste de la dynamique, d'ajuster le paramètre de dynamique à partir de données issues de plusieurs milieux orientants dont les tenseurs d'alignement sont suffisamment différents les uns des autres.

Ainsi, nous combinerons les données provenant de $m$ milieux d'alignement différents, pour extraire le paramètre de dynamique $(S, \sigma$ ou $\theta)$ du résidu $r$. Dans ce dessein, nous utiliserons la méthode des moindres carrés qui consiste à minimiser la fonction [36]:

$$
\chi_{r}^{2}(\{S, \sigma, \theta\})=\sum_{m}\left(\frac{D_{r, m}^{\exp }-\left\langle D_{r, m}^{\text {calc }}\right\rangle}{\sigma_{r, m}}\right)^{2}
$$

où $D_{r, m}^{\text {exp }}$ est le couplage dipolaire expérimental du résidu $r$ dans le milieu $m, D_{r, m}^{c a l c}$ est le couplage dipolaire du même résidu calculé par la formule du modèle qui nous intéresse et $\sigma_{r, m}$ l'erreur relative à la mesure du couplage de ce résidu.

Il faut bien noter la différence majeure entre les deux fonctions $\chi^{2}(35,36)$ définies dans ce paragraphe. Alors que l'équation 35 se calcule avec tous les couplages d'un même milieu, l'équation 36, elle, ne prend en compte que les couplages relatifs à un unique résidu provenant des différents milieux d'alignement.

Le calcul de l'incertitude est effectué à l'aide de simulations Monte-Carlo basées sur l'estimation de l'erreur des mesures expérimentales des CDR (la fonction erreur associée aux mesures de CDR est supposée être de type gaussien, la valeur communément appelé « erreur » étant l'écart type de la gaussienne associée).

\section{DÉTERMINATION DE LA MOBILITÉ LOCALE DANS LA PROTÉINE G}

Les trois modèles de dynamique ont été utilisés pour étudier la dynamique de la protéine $\mathrm{G}$ dans une échelle de temps allant de la picoseconde à la milliseconde. Nous disposons des mesures de CDR provenant de cinq milieux orientants différents [21]. Le tenseur d'alignement relatif à chacun des milieux 


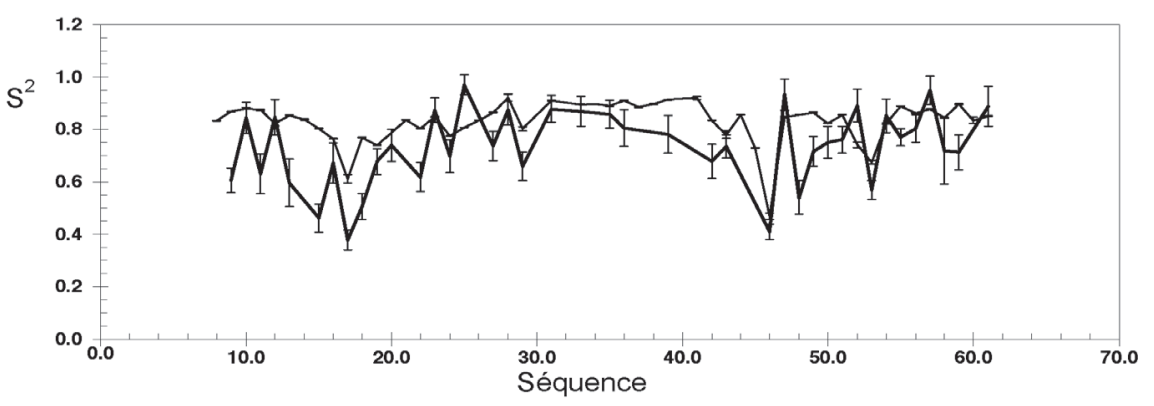

Figure 7. Paramètres de dynamique extraits des CDR (trait foncé) et de la relaxation ${ }^{15} \mathrm{~N}$ (trait fin) de la protéine G. L'erreur est estimée par simulations Monte-Carlo.

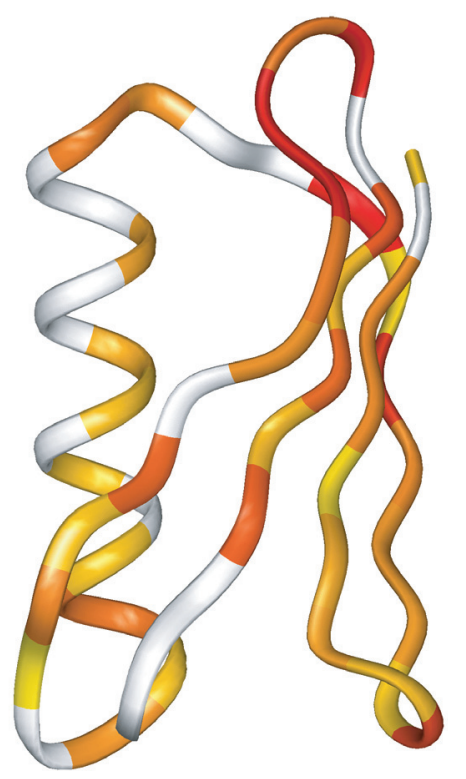

Figure 8. Ruban représentant la structure et la dynamique de la protéine G. En gris clair, les résidus possédant un paramètre d'ordre compris entre 0.75 et 1.0 , en orange entre 0.5 et 0.75 , en rouge entre 0 et 0.5 , en blanc, les résidus dont aucune donnée n'est disponible.

a été déterminé à partir des couplages $\mathrm{C}^{\prime}-{ }^{\alpha} \mathrm{C}$. Le choix d'un des trois modèles pour décrire la dynamique d'un résidu s'est fait d'après les critères décrits précédemment.

Tous les couplages $\mathrm{N}^{\mathrm{N}} \mathrm{H}$ disponibles ont été utilisés pour ajuster le paramètre dynamique de chacun des trois modèles. Le profil du paramètre d'ordre $S^{2}$, dérivé pour chaque résidu du modèle le plus adapté, est montré dans la figure 7. Nous y avons ajouté le profil du paramètre d'ordre extrait de la relaxation ${ }^{15} \mathrm{~N}$ (relatif aux mouvements de l'ordre de la picoseconde à la nanoseconde) [37].

Nous observons une excellente corrélation entre les paramètres d'ordre issus des l'analyses des CDR et des paramètres de relaxation pour la protéine G. Dans l'hélice $\alpha$, le $S^{2}$ issu des CDR est similaire à celui issu de la relaxation. Nous en déduisons qu'il n'y a pas ou peu de mouvements additionnels jusqu'à la milliseconde dans l'hélice $\alpha$. Néanmoins, dans plusieurs sites, des mouvements additionnels, par rapport à ceux qui sont décrits par la relaxation, sont nécessaires pour expliquer la valeur des CDR mesurés. Dans tous ces sites, la différence des résultats issus des CDR et de la relaxation suggère la 
présence d'une activité dynamique qui se situe dans l'échelle de temps uniquement accessible aux CDR, c'est-à-dire de la nano- à la milliseconde.

Aucun résidu ayant une dynamique mieux décrite par le modèle du saut à deux sites n'a été trouvé. Les deux modèles, FAG et à symétrie axiale, sont optimaux dans approximativement la moitié des sites. Aucune corrélation évidente n'a été trouvée entre le modèle sélectionné, la topologie ou l'amplitude de la dynamique.

\section{CONCLUSIONS}

La spectroscopie RMN est un outil puissant pour l'étude des mobilités locales d'une protéine. Parallèlement aux études sur la relaxation de spin, l'utilisation des couplages dipolaires résiduels apporte des informations nouvelles: d'abord, sur les mouvements lents (de la micro- à la milliseconde); ensuite, sur la topologie du mouvement par l'introduction de modèles géométriques.

\section{Références}

[1] Palmer III A.G., Williams J., and McDermott A., J. Phys. Chem. 100 (1996) 13293-13310.

[2] Fischer M.W.F., Majumdar A. and Zuiderweg E.R.P., Progress in NMR Spect. 33 (1998) 207-272.

[3] Lipari G. and Szabo A., J. Am. Chem. Soc. 104 (1982) 4546-4559.

[4] Lipari G. and Szabo A., J. Am. Chem. Soc. 104 (1982) 4559-4570.

[5] Palmer III A.G., Kroenke C. and Loria J.P., Methods in Enzymology, 339 (2001) 204-238.

[6] Tjandra N. and Bax A., Science 278 (1997) 1111-1114.

[7] Tolman J.R., Flanagan J.M., Kennedy M.A. and Prestegard J.H., Proc. Natl. Acad. Sci. 92 (1995) 9279-9283.

[8] Prestegard J.H., Al-Hashimi H.M. and Tolman J.R., Quart. Rev. Biophys. 33 (2000) 371-424.

[9] Bax A., Kontaxis G. and Tjandra N., Methods in Enzymology 339 (2001) 127-174.

[10] de Alba E. and Tjandra N., Prog. Nucl. Magn. Reson. Spectrosc. 40 (2002) 175-197.

[11] Tolman J.R., Curr. Opin. Struct. Biol. 11 (2001) 532-539.

[12] Tolman J.R., Flanagan J.M., Kennedy M.A. and Prestegard J.H., Nature Struct. Biol. 4 (1997) 292-297.

[13] Bax A. and Tjandra N., Nature Struct. Biol. 4 (1997) 254-256.

[14] Fischer M.W.F., Losonczi J.A., Weaver J.L. and Prestegard J.H., Biochemistry 38 (1999) 9013-9022.

[15] Tian F.F., Al-Hashimi H.M., Craighead J.L. and Prestegard J.H., J. Am. Chem. Soc. 123 (2001) 485-492.

[16] Tolman J.R., Al-Hashimi H.M., Kay L.E. and Prestegard J.H., J. Am. Chem. Soc. 123 (2001) 1416-1424.

[17] Meiler J., Prompers J.J., Peti W., Griesinger C. and Brüschweiler R., J. Am. Chem. Soc. 123 (2001) 6098-6107.

[18] Peti W., Meiler J., Brüschweiler R., and Griesinger C, J. Am. Chem. Soc. 124 (2002) 5822-5833.

[19] Tolman J.R., J. Am. Chem. Soc. 124 (2002) 12020-12030.

[20] Briggman K.B. and Tolman J.R., J. Am. Chem. Soc. 125 (2003) 10164-10165.

[21] Ulmer T.S., Ramirez B.E., Delaglio F. and Bax A., J. Am. Chem. Soc. 125 (2003) 9179-9191.

[22] Ernst R.R., Bodenhausen G. and Wokaun A., Principles of Nuclear Magnetic Resonance in One or Two Dimensions. Claredon Press, Oxford (1987).

[23] Levitt H.M., Spin Dynamics: Basics of Nuclear Magnetic Resonance. Wiley, Chippenham (1988).

[24] Saupe A., Angew. Chem. Internat. Edit. 7 (1968) 97-112.

[25] Ramirez B.E. and Bax A., J. Am. Chem. Soc. 120 (1998) 9106-9107.

[26] Prestegard J.H. and Kishore A.I., Curr. Opin. Chem. Biol. 5 (2001) 584-590. 
[27] Sass J., Cordier F., Hoffman A., Rogowski M., Cousin A., Omichinski J.G., Löwen H. and Grzesiek S., J. Am. Chem. Soc. 121 (1999) 2047-2055.

[28] Skrynnikov N.R., Goto N.K., Yang D., Choy W.-Y., Tolman J.R., Mueller G.A. and Kay L.E., Orienting L.E., J. Mol. Biol. 295 (2000) 1265-1273.

[29] Bernadó P. and Blackledge M., J. Am. Chem. Soc. 126 (2004) 4907-4920.

[30] Brüschweiler R. and Wright P.E., J. Am. Chem. Soc. 116 (1994) 8426-8427.

[31] Bremi T. and Brüschweiler R., J. Am. Chem. Soc. 119 (1997) 6672-6673.

[32] Lienin S.F., Bremi T., Brutscher B., Brüschweiler R. and Ernst R.R., J. Am. Chem. Soc. 120 (1998) 9870-9879.

[33] Fischer M.W.F., Zeng L., Pang Y., Hu W., Majumdar A. and Zuiderweg E.R.P., J. Am. Chem. Soc. 119 (1997) 12629-12642.

[34] Pang Y. and Zuiderweg E.R.P., J. Am. Chem. Soc. 122 (2000) 4841-4842.

[35] Dosset P., Hus J.-C., Marion D. and Blackledge M., J. Biomol. NMR 20 (2001) 223-231.

[36] Bernadó P. and Blackledge M., J. Am. Chem. Soc. 126 (2004) 7760-7761.

[37] Hall J.B. and Fushman D., J. Biomol. NMR 27 (2003) 261-275. 